\title{
BMJ Open Patient safety in transitional care of the elderly: effects of a quasi-experimental interorganisational educational intervention
}

Marianne Storm, ${ }^{1}$ Jörn Schulz, ${ }^{2}$ Karina Aase ${ }^{1,3}$

To cite: Storm M, Schulz J, Aase K. Patient safety in transitional care of the elderly: effects of a quasi-experimental interorganisational educational intervention. BMJ Open 2018;8:e017852. doi:10.1136/ bmjopen-2017-017852

- Prepublication history for this paper is available online. To view these files, please visit the journal online (http://dx.doi org/10.1136/bmjopen-2017017852).

Received 22 May 2017 Revised 6 November 2017 Accepted 10 November 2017
Check for updates

${ }^{1}$ Faculty of Health Sciences, Centre for Resilience in Healthcare, University of Stavanger, Stavanger, Norway ${ }^{2}$ Department of Research, Helse Stavanger HF, Stavanger, Norway

${ }^{3}$ Regional Centre for Age-related Medicine and Coordination, Stavanger University Hospital, Stavanger, Norway

Correspondence to Professor Marianne Storm; marianne.storm@uis.no

\section{ABSTRACT}

Objective The study objective was to assess the effects of an interorganisational educational intervention called the 'Meeting Point' on patient safety culture among staff in hospital and nursing home wards.

Design The study employs a quasi-experimental, non-randomised design with a hospital and nursing home intervention group and a hospital and nursing home control group. The study uses one preintervention and two postintervention survey measurements. The intervention group participated in an educational programme 'The Meeting Point' including interorganisational staff meetings combining educational sessions with a discussion platform focusing on quality and safety in transitional care of the elderly.

Results The results show a stable development over time for the patient safety culture factor 'Handoff and transitions', and small improvements for 'Overall perceptions of patient safety culture' and 'Organisational learning - continuous improvement' for the hospital intervention group. No similar development was reported in the nursing home intervention group, which is most likely explained by ongoing organisational changes. Qualitative data show the existence of ongoing initiatives in the hospital to improve transitional care, but not all were connected to the 'Meeting Point'.

Conclusion The 'Meeting Point' has the potential to be a useful measure for healthcare professionals when aiming to improve patient safety culture in transitional care. Further refinement of the key components and testing with a more robust study design will be beneficial.

\section{INTRODUCTION}

Transitional care, including patient handovers and care transitions, can be seen as the actions designed to ensure coordination and continuity of care as patients transfer across different levels of care and/or locations (eg, from a hospital to a nursing home) or between units of care within the same location. ${ }^{1}$ Care transitions represent a vital risk to vulnerable elderly patients in need of multiple healthcare services due to possible breaches in information exchange, responsibility, competence, organisation of work

\section{Strengths and limitations of this study}

- The study is the first to assess the effects of an interorganisational educational programme on assessments of patient safety culture among staff in hospital and nursing home wards.

- A strength is the mixed methods design with the qualitative data collection providing information on contextual factors and measures to improve transitional care in the hospital and nursing home wards, with a possible influence on the reports on patient safety culture.

- There was a stable development over time for the safety culture factor 'Handoff and transitions', and small improvements for 'Overall perceptions of patient safety culture' and 'Organisational learning - continuous improvement' for the hospital intervention group. However, based on the qualitative data we cannot conclude that the improvements were solely connected to the 'Meeting Point'.

- Sample size estimates were conducted to measure the effects of the intervention. The study was limited by the relatively small nursing home intervention group, which also underwent some structural changes during the study period.

tasks, and involvement of patients and nextof-kin. ${ }^{23}$ As such, patient handovers and care transitions have become a focused area of healthcare delivery worldwide. ${ }^{45}$

Transitional care has links to patient safety culture, as cultural composites in both hospital and nursing homes include handoffs and transitions. ${ }^{6} 7$ Handoffs and transitions are operationalised as transfers of patient care information across units of care and during shift changes, when care plans change or when patients are transferred between a hospital and nursing home. Little is known about how improvements in transitional care affect staff perceptions of patient safety culture in their respective hospital or nursing home wards. Patient safety culture can contain interorganisational features in 
addition to being a factor of patient safety culture in the hospital and nursing home setting. Staff in hospital and nursing homes in one geographical area share care responsibilities and care practices for older patients moving across levels of care, and they collaborate to organise good care processes. ${ }^{8}$ Therefore, improvements in interorganisational patient safety culture may be one way to improve transitional care.

Reports on cross-level (eg, between hospital and nursing home) and cross-unit (eg, between hospital wards) patient safety culture measures are lacking in the literature. So far, most strategies have been concerned with singleorganisation measures, such as leadership walk rounds, team-based strategies and training programmes. ${ }^{9}$ Engaging staff in interorganisational learning collaboratives has been reported useful in changing organisational practices and improving the quality of care process. ${ }^{10}$ Discussion platforms and job rotation are also measures suggested for improving transitional care. ${ }^{11} 12$ It is also argued that educational interventions offered to staff in interorganisational settings are particularly relevant to reflect the functional whole of stakeholders involved in transitional care and the complexity of transitional care, fostering a common understanding and responsibility for patient care during care transition situations. ${ }^{13-16}$

In an observational study of 41 transitional care situations (hospital admission and discharge) of older patients, we identified several challenges to quality in transitional care and suggested intraorganisational staff meetings as one useful improvement measure. ${ }^{3}$ We designed an educational intervention called the 'Meeting Point' including interorganisational staff meetings with staff across care levels (hospital and nursing home) and across care units (hospital emergency, medical wards) within the same hospital location. ${ }^{17}$ In this article, we report results from a quasi-experimental study assessing the effects of the 'Meeting Point' on patient safety culture among staff in hospital and nursing home wards. The 'Meeting Point' combines educational sessions with a discussion platform with the objective of improving staff perceptions of hospital and nursing home patient safety culture, and their competencies in patient safety in transitional care.

In this article, we hypothesise the following:

Participation in an educational intervention programme for transitional care has a positive effect on healthcare staff's perception of patient safety culture in the hospital and nursing home wards (H1).

To assess possible short-term and long-term effects of the 'Meeting Point' intervention on staff perceptions of patient safety culture, we included one preintervention and two postintervention measurements, one directly after the 'Meeting Point' and one after 12 months. A qualitative component was included to gather insight into the contextual factors that could influence reports of patient safety culture and to identify ongoing measures to improve transitional care following the 'Meeting Point' intervention.

\section{METHODS}

\section{Study design and setting}

The study design includes a quantitative and a qualitative component and can be described as a concurrent embedded mixed method study. ${ }^{18}$ To assess the effects of the 'Meeting Point', the study employs a quasiexperimental, non-randomised design with a hospital and nursing home intervention group, along with a hospital and nursing home control group, including three quantitative survey measurements on patient safety culture. A qualitative component positioned after the 'Meeting Point' involved data collection at follow-up meetings with staff in the hospital and nursing home wards.

The study was conducted between September 2013 and February 2015. It involved a city-based university hospital in the Western Norwegian Regional Health Authority and three nursing homes in the hospitals' belonging city (population approximately 128000 ). Five hospital wards at the city-based university hospital were included in the study (three wards in the medical division and two wards in the emergency department (ED)). Nursing home wards from three different nursing homes in the city were included.

The study wards were selected based on their similarity in terms of number of staff and ward type and included based on the ward leaders' interest in participation, using one preintervention and two postintervention survey measurements. One medical hospital ward, one emergency ward and two nursing home wards were assigned to the intervention group based on leader and staff willingness to participate at the 'Meeting Point'. Two medical hospital wards, one ward of the ED, and short-term and long-term wards in one nursing home participated as a control group. Table 1 presents the characteristics of the intervention group and control group wards.

\section{Quantitative data collection}

Healthcare professionals in the intervention and control groups received a pretest survey measurement (T1) in September 2013 prior to the intervention, and a post-test survey measurement (T2) in February 2014 immediately after the educational intervention programme. A 12-month follow-up survey measurement (T3) was conducted in February 2015 in order to assess long-term effects of the intervention on patient safety culture perceptions among hospital and nursing home professionals. Sample sizes were estimated using power analysis. We hypothesised that the intervention group's reporting of patient safety culture would improve by $10 \%$ following participation in the intervention. The required sample size for the two-sample t-test comparisons of the means (with a power of $80 \%$ ) was estimated to be 75 people for the intervention group and 150 people for the control group. Thus, a total sample size of 225 people was targeted. ${ }^{17}$ 


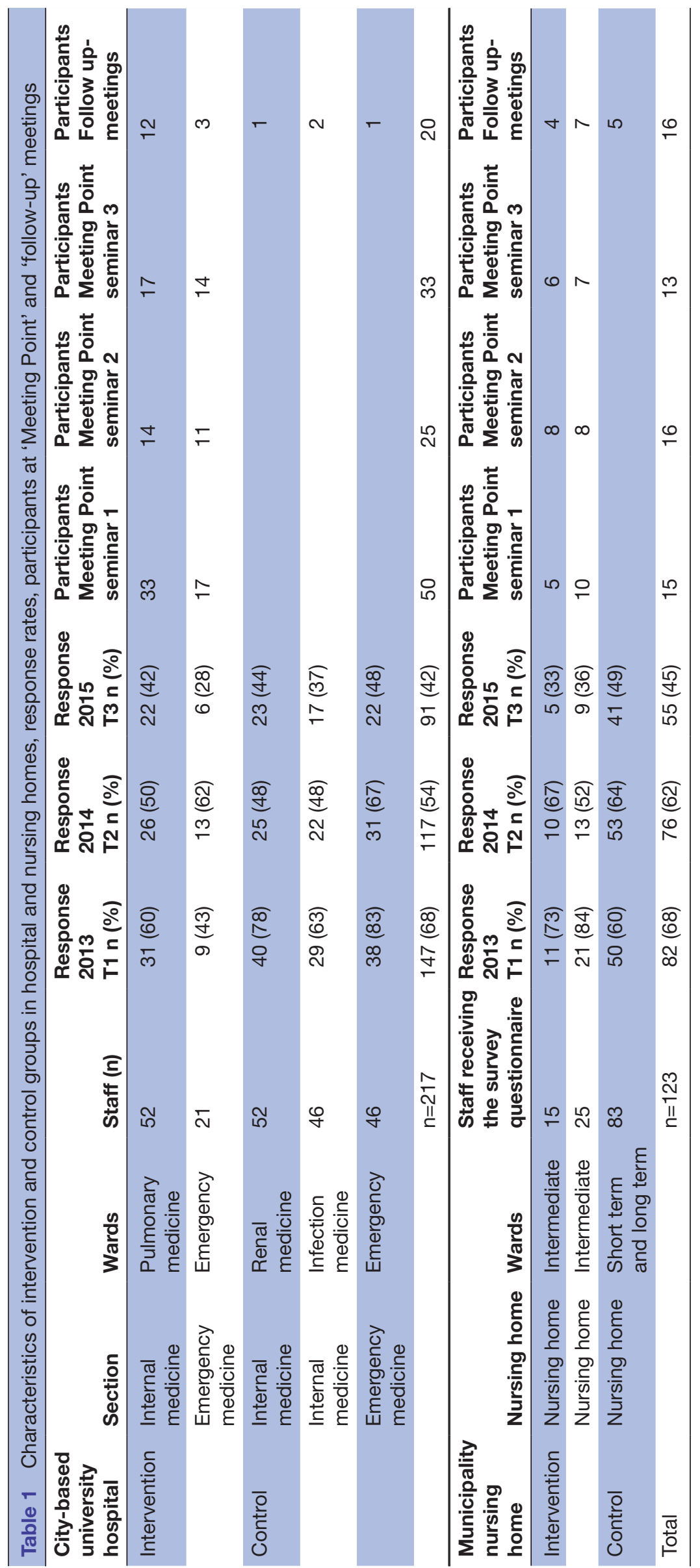




\section{Study participants}

Study participants were healthcare professionals, including nurses, nursing assistants, medical doctors and ward leaders. The intervention and control groups were selected based on their similarity in terms of number of staff and ward type. The healthcare professionals in the hospital intervention group were employed in the medical ward for pulmonary diseases and in the emergency room of the ED. The hospital control group included healthcare professionals working in the renal disease medical ward, the infection medicine ward and one ward of the ED. In the municipality, healthcare professionals in the intervention group were employed in intermediate care wards, at two nursing homes in the municipality. The control group included healthcare professionals from short-term and long-term wards in one nursing home. ${ }^{17}$

\section{Interorganisational educational intervention programme, the 'Meeting Point'}

The interorganisational educational intervention, the 'Meeting Point', was carried out between September 2013 and January 2014. The 'Meeting Point' was conducted as half-day seminars organised by a research team of nine members. The 'Meeting Point' consisted of an educational sessions and a discussion platform including participants from different professions, working across levels (hospital and nursing homes) and units of care (different hospital and nursing home wards). There were also participants from home care services, patient coordination offices in the municipality and administrative personnel in the hospital, to ensure presence from all relevant units involved in care transitions. However, they did not take part in the surveys on patient safety culture. ${ }^{17}$ The number of participants at the 'Meeting Point' seminars from the hospital intervention and nursing home wards is displayed in table 1. Three thematic areas related to transitional care were addressed: (1) risk factors, (2) patient perspectives and (3) system perspectives. Each seminar had a $15 \mathrm{~min}$ introduction, followed by a $45 \mathrm{~min}$ teaching session conducted by the members of the research team. One member of the research team introduced a scenario specific to the thematic area (eg, a text-based case of risk factors in transitional care, a film scenario representing the patient perspective in transitional care and a film illustrating the system perspective) to the participants, followed by group activities facilitated by the members of the research team. The groups were mixed to ensure that they were interprofessional and included participants working in different hospital and nursing home wards. The groups addressed two to three questions related to the scenarios and then identified possible measures to improve transitional care at the ward level. Each 'Meeting Point' seminar ended with the participants taking 5 min to provide their written evaluation. Table 2 outlines the elements, period, contents and purpose of the 'Meeting Point'.
The study protocol 'Quality and safety in the transitional care of the elderly (phase 2)' provides the full details of the intervention programme. ${ }^{17}$

\section{Outcome measures}

Common and widely used measures for patient safety culture are the Hospital Survey on Patient Safety Culture (HSOPSC) and the Nursing Home Survey on Patient Safety Culture (NHSOPSC). ${ }^{619} 20$ Both measures have been developed by the Human Services Agency for Healthcare Research and Quality, US Department of Health and Human Services, and focus on a range of factors including handoff and transitions, organisational learning, teamwork, communication openness and management support for patient safety. The original 12-factor structure of the HSOPSC has been replicated across two measurements with the same sample of Norwegian healthcare professionals. ${ }^{21} 22$ A Cronbach's $\alpha$ of 0.51 was reported for the factor 'Organisational learning - continuous improvement', while ranging between 0.64 and 0.82 for the remaining factors. The NHSOPSC has been validated in the Norwegian nursing home setting reporting a 10-factor solution. ${ }^{23}$ The Cronbach's $\alpha$ values for the factors 'Staffing' and 'Compliance with procedures' were 0.55 and 0.58 , respectively, and the values for the remaining factors ranged from 0.65 to 0.90 .

\section{Statistical methods}

The statistical software package SPSS V.23 was used for statistical data analysis. The internal consistency (reliability) for both the HSOPSC and NHSOPSC factors was measured by Cronbach's $\alpha$ coefficients. Descriptive analyses were performed to illustrate sample characteristics. A Pearson $\chi^{2}$ test was used to compare proportions of categorical variables between healthcare professionals in the intervention group and the control group. Negatively worded items were recoded/ reversed to ensure that higher scores indicate a better safety culture. ${ }^{1920} \mathrm{~A}$ mean sum score was calculated for each of the factors in the HSOPSC and the NHSOPSC across the three measurements. In addition, mean score differences between premeasurements and postmeasurements were calculated and tested with a MannWhitney U test.

A multiple regression analysis using hierarchical mixed linear models (MLM) ${ }^{24}$ is used to describe the effects of the 'Meeting point' on the safety culture factors. An unstructured correlation matrix is assumed because of the unequal distances between the repeated responses. MLM assumptions are checked prior to the analyses, for example, normality in the residuals using Q-Q plots. The study uses a similar analytical approach as Haugen et al. ${ }^{25}$ Non-responders can be a problem when conducting studies with repeated measurements. The MLM is beneficial as it includes participants responding only to one or two of the measurements in the analyses. Each of the patient safety culture factors was included 
Table 2 The elements, period, contents and purpose of the 'Meeting Point' based on Storm et al ${ }^{17}$

\begin{tabular}{|c|c|c|c|}
\hline Elements & $\begin{array}{l}\text { Approximate } \\
\text { time (min) }\end{array}$ & Contents & Purpose \\
\hline $\begin{array}{l}\text { Introduction by members of the } \\
\text { research team }\end{array}$ & 15 & $\begin{array}{l}\text { Seminar 1: project presentation } \\
\text { 'Quality and safety in transitional care } \\
\text { of the elderly' } \\
\text { Seminars } 2 \text { and } 3 \text { : welcome and } \\
\text { summary of previous seminar, } \\
\text { participant evaluations and identified } \\
\text { measures }\end{array}$ & $\begin{array}{l}\text { Introduce the participants to the } \\
\text { research project, its main purpose } \\
\text { and status } \\
\text { Keep focus during the intervention } \\
\text { and track of recent activities at the } \\
\text { Meeting Point and in the wards }\end{array}$ \\
\hline $\begin{array}{l}\text { Teaching sessions held by one } \\
\text { or several of the research team } \\
\text { members }\end{array}$ & 45 & $\begin{array}{l}\text { Three thematic areas: } \\
\text { Risk factors } \\
\text { Patient perspective } \\
\text { System perspective }\end{array}$ & $\begin{array}{l}\text { Increase competencies of quality and } \\
\text { safety in transitional care of elderly } \\
\text { Introduce evidence and tools to } \\
\text { improve quality in transitional care }\end{array}$ \\
\hline $\begin{array}{l}\text { Scenarios developed by the } \\
\text { research team and the regional } \\
\text { health authority }\end{array}$ & 15 & $\begin{array}{l}\text { Text-based patient case of risks } \\
\text { factors } \\
\text { Film illustrating the patient and } \\
\text { system perspective }\end{array}$ & $\begin{array}{l}\text { Focus attention to the three thematic } \\
\text { areas to stimulate individual reflection } \\
\text { and group activity }\end{array}$ \\
\hline $\begin{array}{l}\text { Plenary discussion led by } \\
\text { members of the research team }\end{array}$ & 45 & $\begin{array}{l}\text { Group presentations of improvement } \\
\text { measures identified and agreed on }\end{array}$ & $\begin{array}{l}\text { Discussion and agreement of } \\
\text { measures for implementation at the } \\
\text { wards }\end{array}$ \\
\hline Evaluation & 5 & $\begin{array}{l}\text { Five-item questionnaire: if the } \\
\text { 'Meeting Point' had fulfilled } \\
\text { expectations, been beneficial to own } \\
\text { clinical work, if patient cases were } \\
\text { relevant and if anything should be } \\
\text { revised }\end{array}$ & $\begin{array}{l}\text { Written feedback from participants } \\
\text { on the key components of the } \\
\text { educational programme and } \\
\text { experiences with interorganisational } \\
\text { staff meetings }\end{array}$ \\
\hline
\end{tabular}

as dependent variable and associated with the independent variables: group (control or intervention); survey (T1, T2 or T3); and an interaction term between group and survey. The variable group measures the population average difference of safety factors between intervention and control groups, whereas the variable survey reflects the effect of measurement time with pretest survey measurement $\mathrm{T} 1$ as the reference group. ${ }^{\mathrm{i}}$ The

\footnotetext{
${ }^{\mathrm{i}}$ The hierarchical model can be described by $\mathrm{Y}=\beta \_0+\beta \_1 \times$ Group $+\beta \_2 \times-$ Survey_1+ $\beta \_3$ Survery_2+ $\beta \_4 \times$ Group $\times$ Survey_1+ $\beta \_5 \times$ Group $\times$ Survey_2. $\beta \_0$ is the intercept (ie, the constant) of the model and $\beta \_1, \ldots, \beta \_5$ are the regression coefficients. Group is $(=0)$ for the control and $(=1)$ for the intervention group, Survey_1 is the independent variable at baseline survey T1 $(=0)$ and postintervention T2 (=1), Survey_2 is the independent variable at baseline survey T1 $(=0)$ and postintervention T3 (=1) and interactions Group $\times$ Survey_1, Group $\times$ Survey_2. The estimated patient safety factors for the control $(\mathrm{CG})$ and intervention group (IG) are $\beta \_0(\mathrm{CG})$ and $\beta \_0+\beta \_1$ (IG) at T1, $\beta \_0+\beta \_2(\mathrm{CG})$ and $\beta \_0+\beta \_1+\beta \_2+\beta \_4$ (IG) at T2, and $\beta \_0+\beta \_3$ (CG) and $\beta \_0+\beta \_1+\beta \_3+\beta \_5$ (IG) at T3. Thus, the change between T1 and T2 is estimated by $\beta \_2$ for the control group and $\beta \_2+\beta \_4$ for the intervention group.
}

analyses were repeated including additional covariates like profession, patient contact and work experience.

\section{Qualitative data collection}

Qualitative data collection took place after the 'Meeting Point' at follow-up meetings between the second (T2) and third survey measurement (T3) to identify measures that had been initiated at the wards related to transitional care and to collect information about ongoing organisational changes (new routines, information technology systems, information exchange and others). In the control group, follow-up meetings with ward leaders and/or nurses were conducted to gain knowledge about the contextual setting and to obtain an overview of potential and ongoing transitional care activities. ${ }^{17}$

Eight follow-up meetings (lasting from 0.5 to 1 hour) were conducted, including 36 participants from the hospital and nursing home intervention and control groups. The follow-up meetings with the intervention group included ward nurses, nurses and auxiliary nurses who had attended the 'Meeting Point' seminars. The 
meeting was organised in connection with lunch hours in the hospital medical ward, as a meeting with the ward nurse and two ED nurses, and in connection with the regular staff meeting in the nursing homes to ensure that staff were able to participate. For the control group, the participants were ward nurses from the hospital wards and one-ward nurse assistants. For the nursing home control group, the nursing home facility manager and four ward nurses took part. The number of participants at the follow-up meetings from the hospital and nursing home wards is displayed in table 1.

Two researchers conducted the data collection: one was responsible for asking questions, while the other took notes and asked follow-up questions. The follow-up meetings were audio-recorded and transcribed verbatim. In the follow-up meetings with the intervention group, a summary of the 'Meeting Point' seminars was given to the participants, and questions related to initiated measures based on the 'Meeting Point' participation, other measures and possible changes at the ward guided the meeting discussion. In the control group meetings, similar questions were raised, except for the reference to the 'Meeting Point'. The analysis of the qualitative data followed a directed content analysis approach to describe the ongoing transitional care activities in the intervention and control groups, specific measures that had been initiated and the organisational context of relevance. ${ }^{26}$

\section{Ethics}

Participation was voluntary and based on informed, verbal and written consent. The leaders of the hospital and nursing home wards received a formal request with information about the study. A meeting between the leader and members of the research team followed this request to inform about the pre-test and post-test measurements and the intervention programme. Staff were informed about the survey measurements and the 'Meeting Point' at personnel meetings held at their respective wards. Recruitment to the control group was conducted in a similar manner, but without introduction of the 'Meeting Point' seminars. All study participants received written information about the project and the measurements together with the safety culture questionnaire.

\section{RESULTS}

\section{Responses to questionnaire surveys}

Three hundred and forty healthcare professionals (217 at the hospital and 123 in nursing homes) were included and received the survey questionnaire. The respondents were given an identification number to ensure they were matched across the three measurements. At T1, there were 229 responses (response rate 67\%); at T2, 193 responses (response rate 54\%); and at T3, 146 responses (response rate $44 \%$ ). At T3, the ward leaders were contacted to provide an overview of professionals not able to respond to the questionnaire. Based on this feedback, 60 professionals were not able to respond to the questionnaire at
T3, as they were not working on the ward anymore, being on sick or maternity leave.

Additional analysis on non-responders in the hospital intervention and control groups revealed a lower proportion of non-responders at $\mathrm{T} 1$ in the control group $(\mathrm{P}=0.005)$ and no difference at T2 and T3. For the nursing homes, there was a lower proportion of non-responders at $\mathrm{T} 1 \quad(\mathrm{P}=0.036)$ in the intervention group compared with the control group but no significant difference at $\mathrm{T} 2$ and T3.

The Pearson $\chi^{2}$ test in table 2 revealed that there was significant difference in proportion for the categorical variables 'profession' $(\mathrm{P}<0.001)$ and 'patient contact' $(\mathrm{P}=0.042)$ for the hospital intervention and control groups. For the nursing home intervention and control groups, there was a significant difference in proportion for the variables 'profession' $(\mathrm{P}=0.028)$ and 'number of years in this nursing home' $(\mathrm{P}<0.001)$. Table 3 presents the characteristics of healthcare professionals responding at $\mathrm{T} 1$.

\section{Reliability of measurement scales}

Reliability was assessed at T1 and T2 for both the Norwegian HSOPSC and NHSOPSC. For the Norwegian 'HSOPSC' $(\mathrm{n}=147)$, the lowest Cronbach's $\alpha$ value was 0.46 ('Communication openness') at T1 but improved to 0.70 at T2. For the remaining factors the Cronbach's $\alpha$ ranged from 0.62 ('Teamwork across units') to 0.83 ('Teamwork within units'), which is acceptable according to Hair et $a l^{27}$ For the NHSOPSC $(\mathrm{n}=82)$, the lowest Cronbach's $\alpha$ values at T1 were 0.50 ('Communication openness'), 0.46 ('Compliance with procedures') and 0.50 ('Nonpunitive response to mistakes'). At T2 Cronbach's $\alpha$ remained low at 0.48 ('Nonpunitive response to mistakes') and 0.50 ('Compliance with procedure'), and decreased to 0.41 for 'Staffing', while improved to 0.66 for 'Communication openness'. Table 4 displays the factors, number of items, factor definition and Cronbach's $\alpha$ at T1 and T2 for the Norwegian versions of HSOPSC and NHSOPSC.

\section{Descriptive statistics and multivariate analyses}

Descriptive statistics analyses (means, SE and 95\% CI) are presented separately for the hospital intervention and control groups at the three measurements in table 5, and for the nursing home intervention and control groups in table 6 .

We calculated the differences between the scores of the premeasurement $\mathrm{T} 1$ to postmeasurement T2 (diff T1-T2) and premeasurement T1 to postmeasurement T3 (diff T1-T3) for the hospital and nursing home intervention and control groups. The Mann-Whitney U test shows a significant difference for the hospital patient safety factors: 'Handoff and transitions' (diff T1-T2) $(\mathrm{P}<0.05)$ and 'Organisational learning - continuous improvement' (diff T1-T3) $(\mathrm{P}<0.05)$. For the three factors 'Overall perceptions of patient safety' and 'Staffing', and the outcome item 'Patient safety grad', significant differences 
Table 3 Characteristics of the healthcare professionals responding to the survey questionnaire at T1, 2013

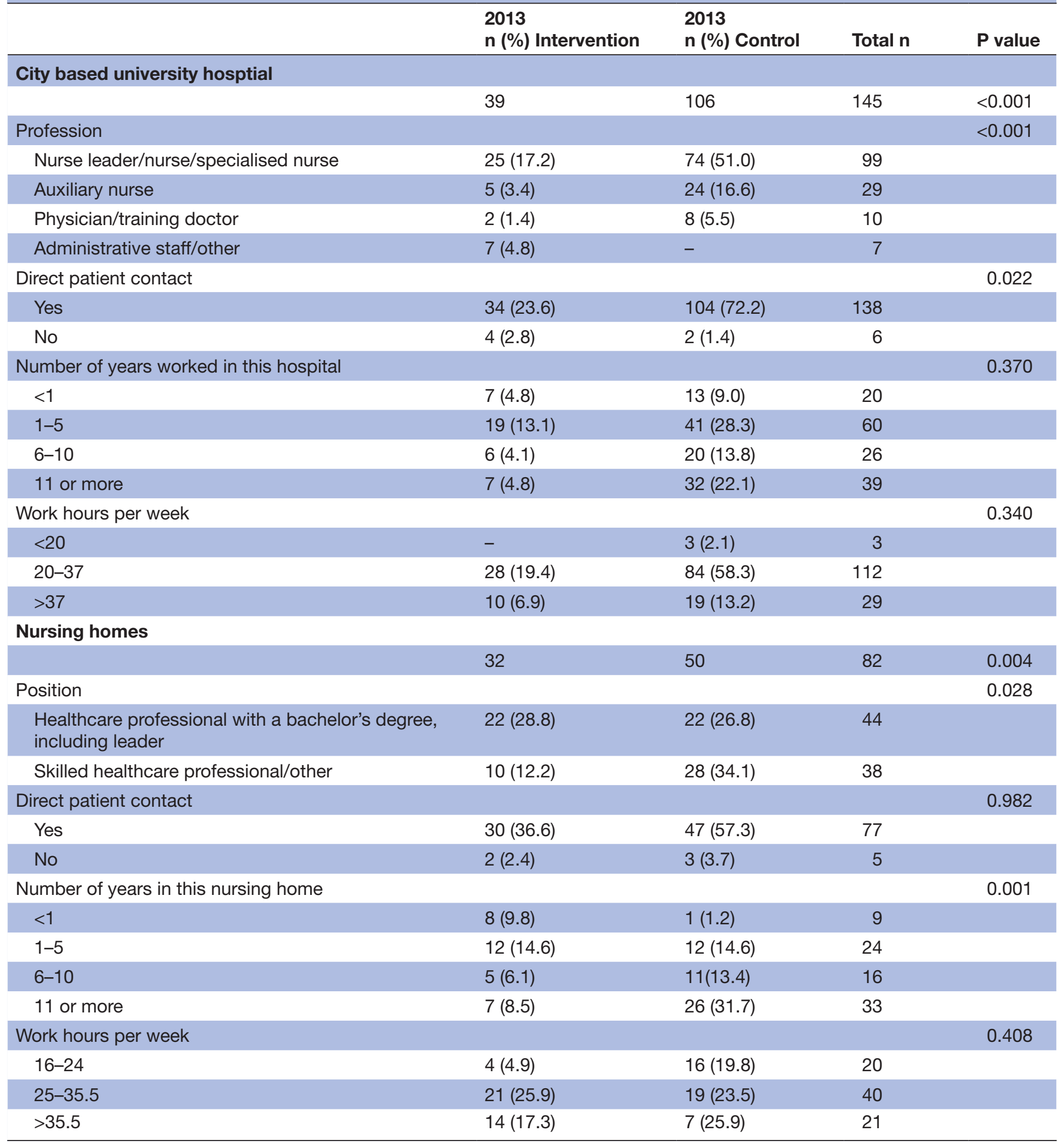

were identified for both time periods, T1-T2 and T1-T3 $(\mathrm{P}<0.05)$. For the nursing home patient safety factors, there was a significant difference for 'Staffing' (diff T1T3) $(\mathrm{P}<0.05)$ and for the overall rating item 'I would tell a friend that this is a safe nursing home for their family' (diff T1-T2) $(\mathrm{P}<0.05)$.

The multivariate analyses with a mixed-model approach (MLM) were conducted for each of the factors in the
HSOPSC and NHSOPSC. All model assumptions were met. Table 7 presents the multivariate analysis with a linear mixed model approach for each of the factors in the HSOPSC. For the hospital intervention and control groups, the MLM reveals a significant intervention effect described by the regression coefficients for interactions between intervention and survey time compared with the control. 
Table 4 The factors and the number of items included in the HSOPSC and NHSOPSC

Cronbach's Cronbach's

Definition: The extent to which...

$\alpha$ (T1) $\quad \alpha$ (T2)

Norwegian version of HSOPSC
12 factors, 42 items and 2 outcome
items

Teamwork within units, 4 items

Teamwork across units, 4 items

Staffing, 4 items

Non-punitive response to errors, 3 items

Handoff and transition, 4 items

Heedback and communication abo

Feedback and communication about
error,3 items
Communication openness, 3 items

Feedback and communication about
error,3 items
Communication openness, 3 items
Staff support each other, treat each other with respect and work together as a team.

0.83

0.84

Hospital units cooperate and coordinate with one another to 0.62 provide the best care for patients.

There are enough staff to handle the workload and work hours 0.63 are appropriate to provide the best care for patients.

Staff feel that their mistakes and event reports are not held

0.73

0.71

against them and that mistakes are not kept in their personnel file.

Important patient care information is transferred across $\quad 0.69$

$0.69 \quad 0.71$

hospital units and during shift changes.

Staff are informed about errors that happen, are given

0.63

0.79

feedback about changes implemented and discuss ways to prevent errors.

Staff freely speak up if they see something that may negatively affect a patient and feel free to question those with more authority.

Supervisor/manager expectations
and actions promoting patient
safety, 4 items

Supervisors/managers consider staff suggestions for improving patient safety, praise staff for following patient safety procedures and do not overlook patient safety problems.

Overall perception of patient safety,

4 items

Management support for patient

safety, 3 items

Procedures and systems are good at preventing errors and there is a lack of patient safety problems.

Hospital management provides a work climate that promotes 0.74

$0.68 \quad 0.74$
patient safety and shows that patient safety is a top priority.

Organisational learning - continuous improvement, 3 items

Mistakes have led to positive changes and changes are evaluated for effectiveness.

$\begin{array}{lll}\begin{array}{l}\text { Frequency of events reported, } 3 \\ \text { items }\end{array} & \text { Mistakes of the following types are reported: (1) mistakes } 0.7 \\ & \text { caught and corrected before affecting the patient, (2) mistakes } \\ & \text { with no potential to harm the patient and (3) mistakes that } \\ & \text { could harm the patient but do not. }\end{array}$

Patient safety grade, 1 item

Number of events reported, 1 item

Norwegian version of NHSOPSC

10 factors, 41 items and 2 outcome items

\begin{tabular}{|c|c|c|c|}
\hline Teamwork, 4 items & $\begin{array}{l}\text { Staff treat each other with respect, support one another and } \\
\text { feel like they are part of a team. }\end{array}$ & 0.78 & 0.71 \\
\hline Staffing, 4 items & $\begin{array}{l}\text { There are enough staff to handle the workload, meet } \\
\text { residents' needs during shift changes and keep residents safe } \\
\text { because there is not much staff turnover. }\end{array}$ & 0.62 & 0.41 \\
\hline $\begin{array}{l}\text { Non-punitive response to mistakes, } \\
4 \text { items }\end{array}$ & $\begin{array}{l}\text { Staff are not blamed when a resident is harmed, are treated } \\
\text { fairly when they make mistakes and feel safe reporting their } \\
\text { mistakes. }\end{array}$ & 0.5 & 0.49 \\
\hline
\end{tabular}

Continued 
Table 4 Continued

Definition: The extent to which...

Cronbach's Cronbach's

$\alpha$ (T1) $\quad \alpha($ (T2)

\begin{tabular}{|c|c|c|c|}
\hline $\begin{array}{l}\text { Feedback and communication about } \\
\text { incidents, } 4 \text { items }\end{array}$ & $\begin{array}{l}\text { Staff discuss ways to keep residents safe, tell someone if } \\
\text { they see something that might harm a resident and talk about } \\
\text { ways to keep incidents from happening again. }\end{array}$ & 0.74 & 0.56 \\
\hline Communication openness, 3 items & $\begin{array}{l}\text { Staff speak up about problems, and their ideas and } \\
\text { suggestions are valued. }\end{array}$ & 0.45 & 0.66 \\
\hline $\begin{array}{l}\text { Supervisor expectations and actions } \\
\text { promoting patient safety, } 3 \text { items }\end{array}$ & $\begin{array}{l}\text { Supervisors listen to staff ideas and suggestions about } \\
\text { resident safety, praise staff who follow the right procedures } \\
\text { and pay attention to safety problems. }\end{array}$ & 0.84 & 0.85 \\
\hline $\begin{array}{l}\text { Management and organisational } \\
\text { learning, } 10 \text { items } \dagger\end{array}$ & $\begin{array}{l}\text { Nursing home management provides a work climate that } \\
\text { promotes resident safety and shows that resident safety is a } \\
\text { top priority. There is a learning culture that facilitates making } \\
\text { changes to improve resident safety and evaluates changes for } \\
\text { effectiveness. Residents are well cared for and safe. }\end{array}$ & 0.9 & 0.89 \\
\hline $\begin{array}{l}\text { Compliance with procedures, } 3 \\
\text { items }\end{array}$ & $\begin{array}{l}\text { Staff follow standard procedures to care for residents and do } \\
\text { not use short cuts to get their work done faster. }\end{array}$ & 0.46 & 0.52 \\
\hline Training and skills, 3 items & $\begin{array}{l}\text { Staff get the training they need, have enough training on how } \\
\text { to handle difficult residents and understand the training they } \\
\text { get in the nursing home. }\end{array}$ & 0.71 & 0.68 \\
\hline
\end{tabular}

Overall patient safety grade, 1 item

Overall safe nursing home, 1 item

*This study uses three 'Handoff' items (originally four) based on the psychometric testing of the Norwegian translated version of the NHSOPSC.

†The three dimensions 'overall perceptions of patient safety' (three items), 'management support for patient safety' (three items) and 'organizational learning' (four items) from the original NHSOPSC have in the Norwegian version been merged to one dimension called 'Management and organizational learning'. ${ }^{23}$

HSOPSC, Hospital Survey on Patient Safety Culture; NHSOPSC, Nursing Home Survey on Patient Safety Culture.

For T1-T2, a significantly different progression in the intervention group is reported on the five patient safety factors 'Handoff and transitions', 0.25 (95\% CI 0.01 to 0.49) $(\mathrm{P}<0.05)$; 'Organisational learning - continuous improvement', 0.29 (95\% CI -0.00 to 0.58$)(\mathrm{P}<0.05)$; 'Overall Perceptions of patient safety', 0.30 (95\% CI 0.03 to 0.57$) \quad(\mathrm{P}<0.05)$; 'Staffing', 0.27 (95\% CI 0.06 to $0.48)(\mathrm{P}<0.05)$; and 'Nonpunitive response to error', 0.24 $(95 \%$ CI 0.00 to 0.49$)(\mathrm{P}<0.05)$. For all five factors, a small increase of $-0.19+0.25=0.06,-0.18+0.29=0.11,-0.20+0.3$ $0=0.10,-0.25+0.27=0.02$ and $-0.09+0.24=0.19$ was identified for T1-T2 in the intervention group, compared with a negative development of $-0.19,-0.18,-0.20,-0.25$ and -0.09 for the control group.

From T1-T3, there was a significant intervention effect compared with the control group on the patient safety factors 'Organisational learning - continuous Improvement' at $0.41(95 \%$ CI 0.06 to 0.76$) \quad(\mathrm{P}<0.05)$; 'Overall perceptions of patient safety' at 0.50 (95\% CI 0.19 to $0.80)(\mathrm{P}<0.01)$; 'Handoff and transitions' at $0.28(95 \% \mathrm{CI}$ 0.02 to 0.53$)(\mathrm{P}<0.05)$; and 'Staffing' at $0.49(95 \% \mathrm{CI}$ 0.19 to 0.79$) \quad(\mathrm{P}<0.01)$. Similar to before, the control group showed a significant decrease in these factors from T1-T3, whereas the intervention group remained stable or gently increased. For instance, for 'Handoffs and Transitions', the safety score remained almost stable at $-0.26+0.28=0.02$ in the intervention group compared with a decrease of -0.28 in the control group.

Furthermore, the MLM analyses in table 7 show, for the intervention group, significantly smaller patient safety culture scores at $\mathrm{T} 1$ for the following factors: 'Handoff and transitions' (-0.43, $\mathrm{P}<0.001)$; 'Organisational learning - continuous improvement' $(-0.45$, $\mathrm{P}<0.001)$; 'Supervisor expectations' $(-0.11, \mathrm{P}<0.05)$; 'Overall perceptions of patient safety' $(-0.53, \mathrm{P}<0.001)$; 'Staffing' $(-0.49, \mathrm{P}<0.001)$; 'Nonpunitive response to error' $(-0.50, \mathrm{P}<0.001)$; and 'Patient safety grade' $(-0.22$, $\mathrm{P}<0.05)$.

In the nursing home wards, the MLM revealed a significant intervention effect for T1-T2 on the item 'I would tell a friend that this nursing home is safe' at $0.20(95 \%$ CI 0.03 to 0.38$) \quad(\mathrm{P}<05)$. For this item, an increase of $-0.04+0.20=0.16$ was identified for T1-T2 in the intervention group compared with -0.04 in the control group. From T1-T3, there were significant intervention effects for 'Teamwork' at $0.52(95 \%$ CI 0.01 to 1.03$)(\mathrm{P}<0.05)$; 'Staffing' at $0.64(95 \%$ CI 0.31 to 0.97$)(\mathrm{P}<0.001)$; and 'Overall rating on patient safety culture' at $0.62(95 \%$ CI 0.07 to 1.17$)(\mathrm{P}<0.05)$. For example, there was a small increase of $-0.58+0.64=0.06$ in the intervention group compared with a decrease of -0.58 in the control group. Table 8 presents the multivariate analysis with a 


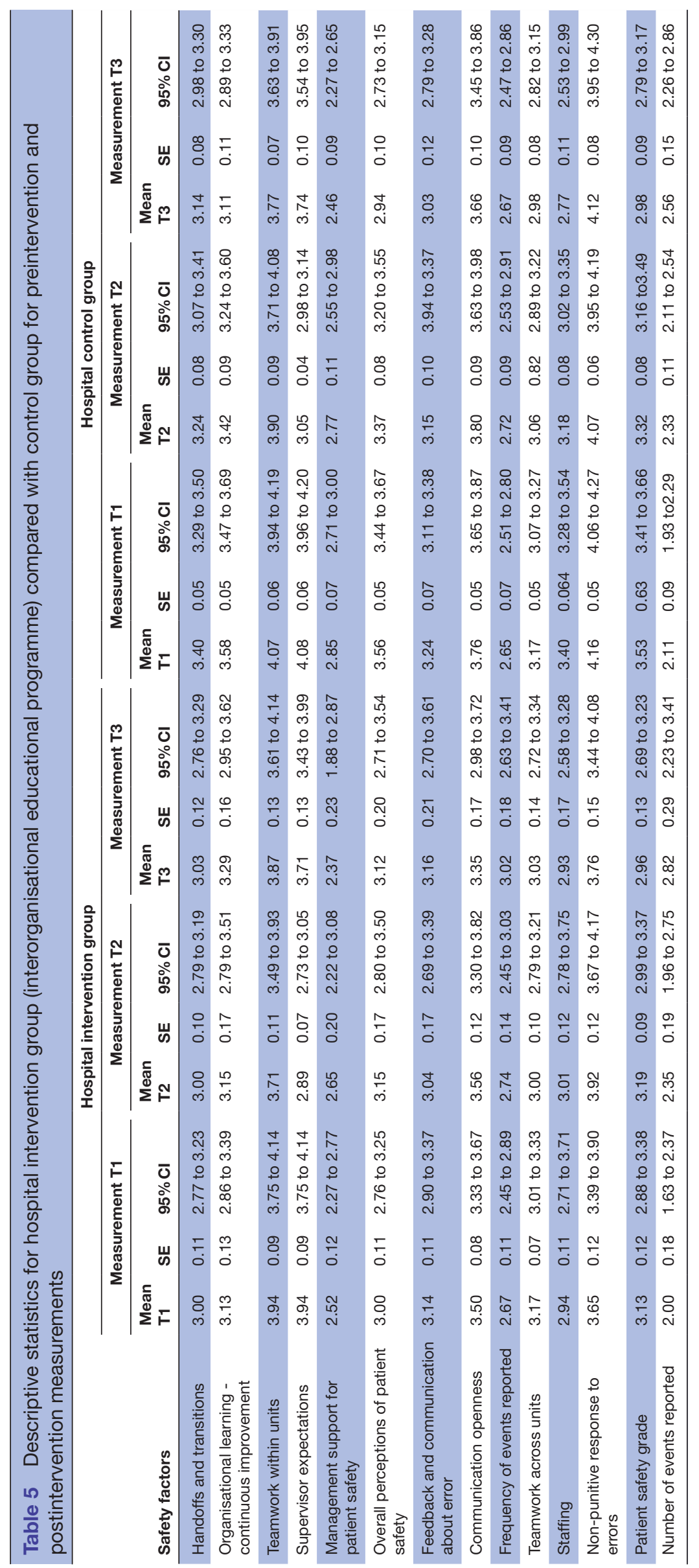




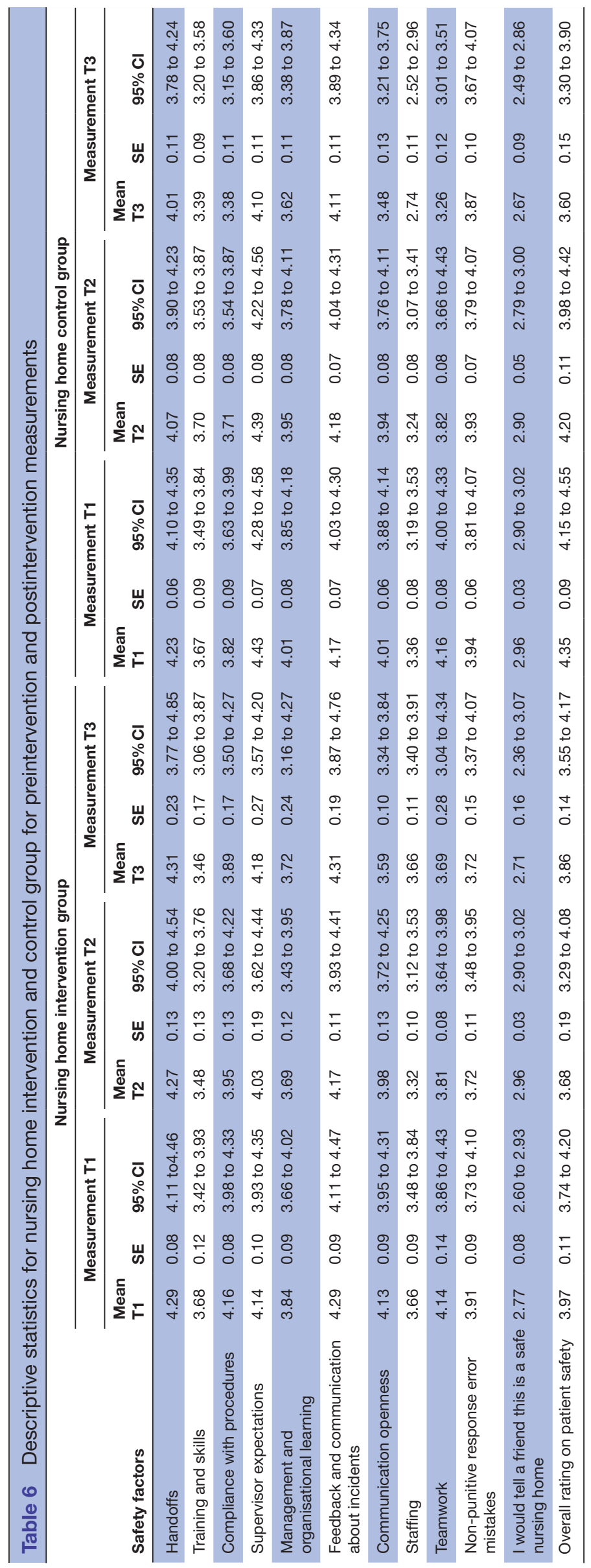




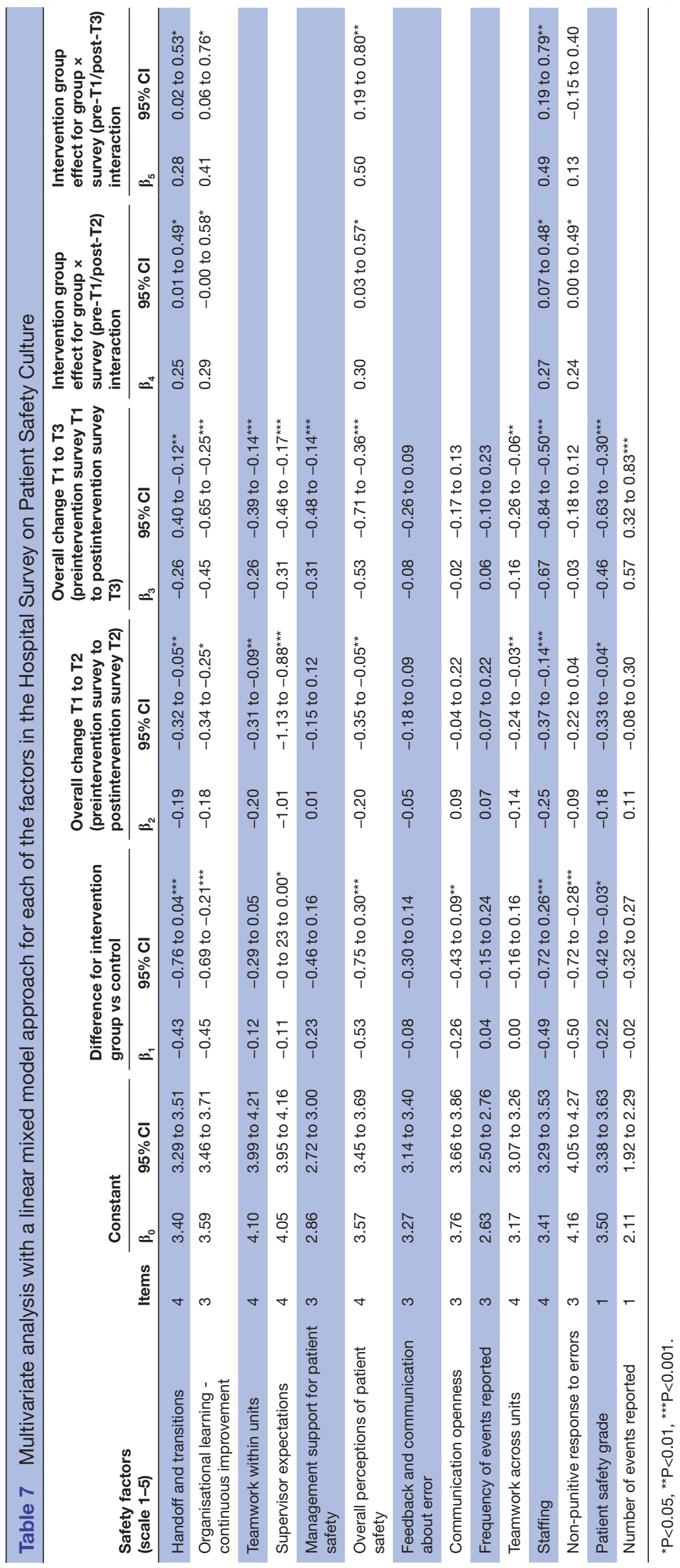




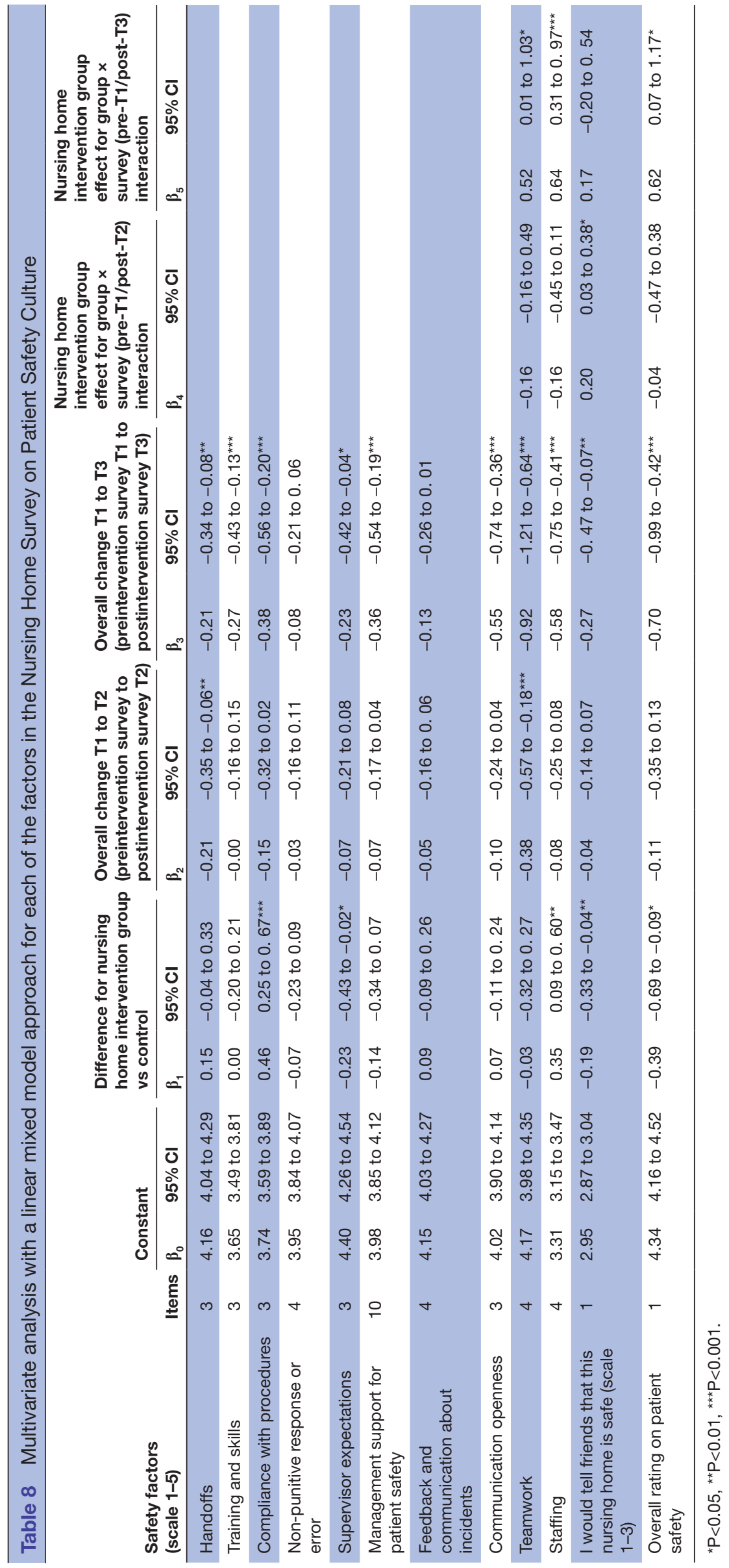


linear mixed model approach for each of the factors in NHSOPSC.

In addition, table 8 reports significantly higher scores at $\mathrm{T} 1 \mathrm{for}$ the intervention group compared with the control group for 'Compliance with procedures' $(\mathrm{P}<0.001)$ and 'Staffing' $(\mathrm{P}<0.05)$, and significantly lower scores for 'Supervisor expectations' $(\mathrm{P}<0.05)$, and for the two single outcome items 'I would tell friends that this nursing home is safe' $(\mathrm{P}<0.05)$ and 'Overall rating on patient safety' $(\mathrm{P}<0.05)$. In the nursing home wards, there was a significant negative development for the control group from T1-T2 for the patient safety factors 'Teamwork' and 'Handoffs'. From T1-T3, there was a significant negative development for most of the patient safety factors.

The MLM analyses adjusted for the additional covariates 'profession', 'work experience' and 'patient contact' resulted in same result patterns.

\section{Ongoing transitional care measures at the wards}

The results from the follow-up meetings are presented separately for the hospital and nursing home intervention and control groups.

\section{Hospital}

In the emergency room of the ED, several initiatives were introduced to improve transitional care between the second and third measurements (T2, T3). One project focused on the overall patient flow in the hospital to avoid overcrowding and was a project initiated as part of a general focus on patient safety in the hospital. This project involved use of a computer-based system that visualised the patient flow, providing a daily report to the leader with information on patient waiting times for the physician (more than 1 hour, more than 3 hours) and expected patient arrivals. The shift leaders had received training to manage the information system. The number of nursing positions had also been increased on evening and night shifts due to several periods of overcrowding. According to the participants, it was not uncommon to have more than 40 patients, with 28 patient beds. Following the 'Meeting Point', two television (TV) screens to provide information on waiting hours had been installed to inform patients.

The internal medicine ward had, according to the participants, about 6-10 discharges per day. The participants mentioned the usefulness of a computer system for electronic messaging of patient information from hospital to municipality, allowing hospital staff to receive information regarding to which nursing home the patient was being discharged. After the 'Meeting Point' a poster visualising the order of messages to be sent to the municipality relating to hospital discharge of patients had come in place.

In the control group, ongoing activities were conducted as part of the National Patient Safety Campaign, addressing issues such as fall prevention, pressure ulcer, nutritional status, urinary infections and central venous line-related infections. Meetings with the hospital control group did not reveal specific initiatives addressing transitional care, but the participants pointed at areas in need of improvement (eg, reconciliation of patient medications, monitoring e-messages between hospital and municipality to avoid delays, and informing patients and the public about transitional care). Challenges in the control group were identified as related to vacant positions and having sufficient and experienced staff on all shifts, particularly during weekends.

\section{Nursing home}

The nursing home intervention group wards were going through organisational changes between survey measurements T2 and T3. The ward leader and nursing staff at the intermediate ward were preparing for the ward to be reorganised into a short-term ward following a decision by the City Council. The number of patient beds was to be reduced, and the leader was establishing new routines for the ward. Similarly, the intermediate nursing home ward had been reorganised, including emergency beds for the municipality health services.

In the nursing home control group, participants referred to a well-established quality system containing procedures and checklists (eg, admissions, fall prevention and so on), including a yearly external quality audit. Another issue of concern was the new budget model for the municipality nursing homes leading to retrenchment measures, such as reduction in level of staffing, cutting the yearly external quality audit and reducing the fulltime position of the quality coordinator. In particular, reducing the level of staffing was said to influence the time available for staff learning and competence development. Recent improvement projects focused on medication reconciliation and preventing pressure ulcers. Electronic information exchange with the hospital was said to have improved over the last year.

\section{DISCUSSION}

This mixed methods study reports results from a quasiexperimental intervention study with an intervention and a control group, an interorganisational educational programme called 'The Meeting Point', to improve patient safety in transitional care of the elderly. Qualitative follow-up meetings reported measures to improve transitional care and contextual factors that could influence on professionals' patient safety culture reports.

Study results show small but significant changes in reports of patient safety culture immediately after the intervention (T2) that continued after 12 months. A gentle increase was reported for 'Organisational learning continuous improvement', 'Overall perceptions of patient safety culture' and a stable development for 'Handoffs and transition' for the hospital intervention group compared with negative development for the control group. No similar development was reported for the nursing home intervention group. For the hospital and nursing home control group, there was a downward development for 
several patient safety factors. The organisational learning - continuous improvement factor contains items that ask if staff perceive that they are doing things actively to improve safety. The activities at the 'Meeting Point', with professionals engaging in group work activities and identifying intervention measures to improve patient safety in transitional care in their own wards, ${ }^{17}$ can be viewed as a first step in organisational improvement. Hoffmann et $a t^{28}$ used a similar approach to improve patient safety culture in general practice, allowing staff to suggest improvement actions in accordance with their own perceived needs.

The mixed methods data collection was valuable and provided insight into improvement measures taking place between T2 and T3 that could have influenced the reports of patient safety culture. Data from the follow-up meetings show that the emergency room used TV screens to provide information to patients about waiting hours and they implemented a daily patient flow registration system. The medical ward also had put into place a poster visualising the order of messages to be sent to the municipality. TV screens and the poster were improvement measures suggested at the 'Meeting Point', but the concurrent implementation of an electronic patient flow registration system might also have contributed to improvement in some patient safety factors in the hospital intervention group. However, the results do indicate a culture fostering organisational learning - continuous improvement and some improvement in information exchange and support for safety improvement among staff. There was an improvement in reports of staffing in the intervention group compared with the control group, and staffing was not an area addressed at the' Meeting Point'. Data from the follow-up meetings revealed that the emergency room had increased their staffing, and that challenges related to vacant positions were present in the hospital control group.

In the nursing homes, there was a significant and overall negative development from T1 to T3 for most of the patient safety culture factors. The qualitative data report that unexpected organisational changes had taken place in the nursing home intervention group between measurements that may have impacted the study results. Follow-up meetings with the nursing home intervention group revealed that staff perceived improvements in information exchange with the hospital; however, there was no significant intervention effect for this factor. Staffing is reported as an area of improvement in most nursing homes. ${ }^{29}$ Although the MLM revealed a small positive effect for staffing, this change cannot be attributed to the 'Meeting Point' intervention. In the nursing home control group, economic constraints were imposed by a new budget model and a reduction in the number of clinical staff.

A further development of the 'Meeting Point' can include full-scale simulation training emphasising interprofessional and interorganisational team training (eg, clinical assessment, information exchange and communication, patient and next-of-kin involvement) in transitional care situations. ${ }^{173031}$ For future evaluation of the 'Meeting Point', including measures at provider and patient levels and more frequent measurements to assess improvement in clinical performance could be useful.

The research team conducted one follow-up meeting between the two postmeasurements. To continue the learning and improvement processes started at the 'Meeting Point' seminars, more involvement and support from the research team, regular reporting and constructive coaching could have fuelled the improvement processes. A systematic review by Nadeem $e t$ al ${ }^{10}$ identified inperson learning sessions, plan-do-study-act cycles, multidisciplinary quality improvement teams, data collection for quality improvement, follow-up calls/email/ web support, organisational/leadership involvement and preplanning groups as important for successful interorganisational learning collaboratives.

\section{Strengths and limitations}

Some threats to causal inference in quasi-experimental designs are tied to the selection of participants, statistical regression, dropout and response bias. ${ }^{32}$ The intervention and control groups were selected based on their similarity in terms of number of staff and ward type and were included in the study based on the ward leaders' willingness to participate. To control for potential preexisting difference between the groups, $\chi^{2}$ statistics with demographic variables were computed, and we included profession, patient contact and work experience as covariates in the MLM to adjust for possible difference. We also conducted analysis of proportion of non-responders.

Hair et $a l^{27}$ argue that a Cronbach value between 0.60 and 0.70 is acceptable. Internal consistency was questionable with alpha values of 0.50 and below for the nursing home patient safety factors 'Compliance with procedures' and 'Nonpunitive response to mistakes' across $\mathrm{T} 1$ and T2, while improved at T2 for 'Communication openness' and was lower for 'Staffing' at T2. Low internal consistency for 'Compliance with procedures' and 'Staffing' has been reported in earlier studies. ${ }^{23} 33$ Low internal consistency can be due to low number of items in the factor, the respondents or aspect relating to construct validity. ${ }^{34}$ Zúñiga $e t a l^{33}$ suggest reformulating and adding items to this factor to 'Compliance with procedures', for example, to include procedures in areas of medication or pressure ulcers relevant to patient safety in nursing homes. As this is the first study applying the Norwegian version of the NHSOPSC in an intervention study to improve patient safety culture, we decided to include all the factors in the statistical analysis. However, we recommend applying the NHSOPSC with a larger study sample to assess the need for refinement.

Sample size estimates were conducted to measure the effects of the intervention. We aimed for, but did not manage to include, 75 participants in the intervention group and 150 in the control group (Storm et $a l^{17}$ ). A particular concern is the low number of participants responding to the surveys at $\mathrm{T} 2$ and $\mathrm{T} 3$ in the nursing home intervention group. The intervention programme 
was conducted over a period of 5 months, with the participants meeting at three half-day seminars in order to minimise the problems of taking staff out of clinical work. Despite these efforts, some study participants did not respond to all the questionnaire surveys or did not attend all three 'Meeting Point' seminars, which is a weakness of the study. The MLM model is beneficial with repeated measurements, as it includes participants responding only to one or two of the measurements.

\section{CONCLUSION}

The results show a stable development over time for the safety culture factor 'Handoff and transitions', and small improvements for 'Overall perceptions of patient safety culture' and 'Organisational learning - continuous improvement' for the hospital intervention group compared with the control group. No similar development was reported in the nursing home intervention and control groups. The qualitative data revealed that the intervention group implemented an electronic patient flow system, which was not connected to the 'Meeting Point', thereby reducing the ability to make strong connections between the intervention and the survey results. We believe that the 'Meeting Point' has the potential to be a useful measure for healthcare professionals when aiming to improve patient safety in transitional care of elderly patients. Further use will require a more robust study design as well as work on the key components of the 'Meeting Point' and measurements. In particular, there seems to be a need to address the impact of educational interventions on patient outcomes.

Acknowledgements The authors express gratitude to the members of the 'Quality and safety in transitional care of the elderly' research team: Dagrunn Nåden Dyrstad, Randi Nisja Heskestad and Kristin A Laugaland from the University of Stavanger. Dyrstad and Heskestad contributed to the data collection in this paper. We express thanks to Allan Øverenget, Kristin Lexow, Martha Therese Gjestsen and Ingelin Testad, staff at the Regional Centre for Age-related Medicine, SESAM at Stavanger University Hospital, who were involved with the research team in the accomplishment of the 'Meeting Point'. The authors also express gratitude to the healthcare professionals who responded to the surveys, and participated in the 'Meeting Point' and in follow-up meetings by sharing their thoughts and experiences.

Contributors MS contributed to the proposal for grants, designed and was responsible for the intervention programme, conducted data collection, performed analyses of quantitative and qualitative data, and prepared and revised the article manuscript. JS provided advice and contributed to the statistical data analyses, and drafting and revising the manuscript. KA developed the proposal for grant application, was project manager for the 'Quality and safety in transitional care of the elderly' research project and contributed in the drafting of the manuscript. All authors read and approved the final manuscript.

Funding The study was funded by Norwegian Research Council (Grant Agreement No 204637).

Competing interests None declared.

Patient consent Not required.

Ethics approval The Western Norway Regional Committee for medical and health research ethics approved the study (REC no. 2011/1978).

Provenance and peer review Not commissioned; externally peer reviewed.

Data sharing statement All data are published as part of this study. Data set is available on request.
Open Access This is an Open Access article distributed in accordance with the Creative Commons Attribution Non Commercial (CC BY-NC 4.0) license, which permits others to distribute, remix, adapt, build upon this work non-commercially, and license their derivative works on different terms, provided the original work is properly cited and the use is non-commercial. See: http://creativecommons.org/ licenses/by-nc/4.0/

(c) Article author(s) (or their employer(s) unless otherwise stated in the text of the article) 2018. All rights reserved. No commercial use is permitted unless otherwise expressly granted.

\section{REFERENCES}

1. Coleman EA, Boult C. American Geriatrics Society Health Care Systems Committee. Improving the quality of transitional care for persons with complex care needs. J Am Geriatr Soc 2003;51:556-7.

2. Laugaland KA, Aase K, Barach $P$. Adressing risk factors for transitional care of the elderly - literature review. In: Albolino B, Bellandi L, Rosal T, eds. Healthcare systems ergonomics and patient safety. London: Taylor\& Francis Group, 2011:183-91.

3. Storm M, Siemsen IM, Laugaland K, et al. Quality in transitional care of the elderly: Key challenges and relevant improvement measures. Int J Integr Care 2014;14:1-15.

4. World Health Organization (WHO). Communication during patient hand-overs patient safety solutions. 2007. http://www.who.int/ patientsafety/solutions/patientsafety/PS-Solution3.pdf

5. World Health Organization (WHO). Assuring medication accuracy at transitions in care. patient safety solutions. Geneva: World Health Organization, 2007;1.

6. Sorra J, Gray J, Famolaro T, et al. AHRQ nursing home survey on patient safety culture: user's guide. 2016. http://www.ahrq. gov/professionals/quality-patient-safety/patientsafetyculture/ nursinghome/index.html

7. Sorra J, Gray L, Steagle L, et al. AHRQ hospital survey on patient safety culture: user's guide. Rockville, MD: Agency for Healthcare Research and Quality, 2016. Retrieved from AHRQ Publication No. 15-0049-EF (Replaces 04-0041).

8. Nedreskår HH, Storm M. Coordination of discharge practices for elderly patients in light of a health care reform. In: Aase K, Schibevaag L, eds. Researching patient safety and quality in health care: a nordic perspective: Taylor \& Francis, 2016

9. Morello RT, Lowthian JA, Barker AL, et al. Strategies for improving patient safety culture in hospitals: a systematic review. BMJ Qual Saf 2013;22:11-18.

10. Nadeem E, Olin SS, Hill LC, et al. Understanding the components of quality improvement collaboratives: a systematic literature review. Milbank Q 2013;91:354-94.

11. Kirsebom M, Wadensten B, Hedström M. Communication and coordination during transition of older persons between nursing homes and hospital still in need of improvement. $J$ Adv Nurs 2013;69:886-95.

12. Kodner DL, Spreeuwenberg C. Integrated care: meaning, logic, applications, and implications-a discussion paper. Int J Integr Care 2002;2:e12.

13. Gordon M, Findley R. Educational interventions to improve handover in health care: a systematic review. Med Educ 2011;45:1081-9.

14. Ryan D, Barnett R, Cott C, et al. Geriatrics, interprofessional practice, and interorganizational collaboration: a knowledge-to-practice intervention for primary care teams. J Contin Educ Health Prof 2013;33:180-9.

15. Stoyanov $\mathrm{S}$, Boshuizen $\mathrm{H}$, Groene $\mathrm{O}$, et al. Mapping and assessing clinical handover training interventions. BMJ Qual Saf 2012;21(Suppl 1):i50-7.

16. Agency for Health Care Research and Quality (AHRQ). Care coordination measures database. 2016. https:// primarycaremeasures.ahrq.gov/care-coordination/

17. Storm M, Groene O, Testad I, et al. Quality and safety in the transitional care of the elderly (phase 2): the study protocol of a quasi-experimental intervention study for a cross-level educational programme. BMJ Open 2014;4:e005962.

18. Curry L, Nunez-Smith M. Mixed methods in health sciences research: a practical primer. Los Angeles: SAGE, 2015.

19. Sorra J, Nieva V. Hospital survey on patient safety culture. Rockville, MD: Agency for Healthcare Research and Quality (AHRQ), 2004.

20. Sorra J, Franklin M, Streagle S. Nursing home survey on patient safety culture. Rockville: Agency for Healthcare Research and Quality, 2008. (Preparedby Westat, under Contract No. 233-02-0087). AHRQ Publication No.08-0060.

21. Olsen E. Safety climate and safety culture in health care and the petroleum industry: psychometric quality, longitudinal change, and 
structural models. PhD thesis UiS. Norway: University of Stavanger, 2009.

22. Olsen $\mathrm{E}$, Aase $\mathrm{K}$. The challenge of improving safety culture in hosptials: a longitudinal study using hospital survey on patient safety culture. Paper presented at the 11th International Probabilistic Safety Assessment and Management Conference and the Annual European Safety and Reliability Conference; 25-29June 2012. Helsinki, Finland: CurranAssociates, Inc.

23. Cappelen K, Aase K, Storm M, et al. Psychometric properties of the nursing home survey on patient safety culture in Norwegian nursing homes. BMC Health Serv Res 2016;16:446.

24. West BT, Welch KB, Galecki AT, et al. Linear mixed models: a practical guide using statistical software. Boca Raton, Fla: Chapman \& Hall/CRC, 2007

25. Haugen AS, Søfteland E, Eide GE, et al. Impact of the World Health Organization's Surgical safety checklist on safety culture in the operating theatre: a controlled intervention study. $\mathrm{Br} J$ Anaesth 2013;110:807-15.

26. Hsieh HF, Shannon SE. Three approaches to qualitative content analysis. Qual Health Res 2005;15:1277-88.

27. Hair J, Black W, Babin B, et al. Multivariate data analysis. 6th edn. New Jersey: Pearson Educational, Inc, 2006.
28. Hoffmann B, Müller V, Rochon J, et al. Effects of a team-based assessment and intervention on patient safety culture in general practice: an open randomised controlled trial. BMJ Qual Saf 2014;23:35-46.

29. Sorra J, Falamoro T, Dyer N, et al. Nursing home survey on patient safety culture: 2011 Comparative database report. Rockville, MD: Agency for Healthcare Research and Quality (AHRQ), 2011.

30. Salas E, Gregory ME, King HB. Team training can enhance patient safety-the data, the challenge ahead. Jt Comm J Qual Patient Saf 2011;37:339-40.

31. Dyrstad DN, Storm M. Interprofessional simulation to improve patient participation in transitional care. Scand J Caring Sci 2017;31:273-84.

32. Cook TD, Campbell DT, Fankhauser G, et al. Quasi-experimentation: design \& analysis issues for field settings. Boston: Houghton Mifflin Co, 1979

33. Zúñiga $F$, Schwappach $D$, De Geest $S$, et al. Psychometric properties of the swiss version of the nursing home survey on patient safety culture. Saf Sci 2013;55:88-118.

34. Tauber KS. The use of cronbach's alpha when developing and reporting research instruments in science education. Res Sci Educ 2016:1-24. 